Questions de communication

\title{
Pour une critique des médias, en cognition située
}

For a critique of media in situated cognition

Divina Frau-Meigs

\section{CpenEdition}

\section{Journals}

Édition électronique

URL : http://journals.openedition.org/questionsdecommunication/7351

DOI : 10.4000/questionsdecommunication.7351

ISSN : 2259-8901

\section{Éditeur}

Presses universitaires de Lorraine

\section{Édition imprimée}

Date de publication : 1 juillet 2007

Pagination : 187-204

ISBN : 978-2-86480-829-9

ISSN : 1633-5961

\section{Référence électronique}

Divina Frau-Meigs, "Pour une critique des médias, en cognition située », Questions de communication [En ligne], 11 | 2007, mis en ligne le 01 juillet 2007, consulté le 12 avril 2021. URL : http:// journals.openedition.org/questionsdecommunication/7351; DOI : https://doi.org/10.4000/ questionsdecommunication.7351 


\section{$>$ ÉCHANGES}

DIVINA FRAU-MEIGS

Centre d'observation des médias des pays anglophones

Université Paris 3-Sorbonne Nouvelle

meigs@wanadoo.fr

\section{POUR UNE CRITIQUE DES MÉDIAS, EN COGNITION SITUÉE}

Résumé. - Cet article est une réponse à l'entretien de Daniel Dayan (Questions de communication, 8, 2005). Par delà l'analyse des médias américains et leur représentation du conflit israelopalestinien, il s'articule autour de trois interrogations : la couverture de la guerre et de la terreur, la réversibilité des images et la médiation journalistique, la relation entre événement et situation. II s'achève par l'examen de ce que la cognition peut apporter comme contribution scientifique au discours de la méthode en sciences de l'information et de la communication. II pose qu'il est essentiel de se confronter à notre existence biologique et médiatique, pour comprendre la véritable nature des médias, à l'interface de la sphère publique et de la sphère privée. La complexité et la plasticité sans cesse renouvelées de la relation média-cerveau permettent de se départir de positions figées et de reconfigurer l'analyse de situations historiques telles que la guerre et la barbarie.

Mots clés. - Sphère publique, événement, situation, cognition, violence, conflit, image, barbarie, médiation, réversibilité, traitement de l'information. 
| es «Échanges » que Questions de communication consacre à «Pour une critique des médias en temps de conflit? »évoque des points importants du travail de l'actualité sur les mentalités. Je voudrais en reprendre quelques-uns, moins pour les critiquer que pour les prolonger par une réflexion qui, si elle est souvent en phase avec les propos de Daniel Dayan (2005), se pose parfois en contre-distinction.

\section{Les médias américains et le conflit israélo-palestinien}

Pour faire écho à la surprise et à l'indignation de Daniel Dayan (2005), je dirai que j'ai éprouvé des sentiments similaires en analysant la façon dont les médias américains traitaient la Palestine. Aux États-Unis, la couverture de la crise israélo-palestinienne penche très nettement en faveur d'Israël, tous médias confondus. Et ce, depuis les années 70, contrairement à la France où des évolutions sont notables et notées par Daniel Dayan. De fait, l'analyse de celui-ci pourrait être reprise terme à terme et inversée, à l'avantage d'Israël. Rémi Brulin, l'un de mes doctorants qui travaille sur la couverture du terrorisme dans le New York Times comme média de référence, fait apparaitre plusieurs points intéressants qui viennent nourrir les idées d'« obfuscation »' et de détournement que Daniel Dayan (2005 : 204) associe aux « pathologies de la lisibilité ». Sans déflorer ses conclusions, il démontre deux dysfonctionnements : l'« obfuscation » quasi complète du point de vue palestinien au public américain ; la présence de deux discours contradictoires des États-Unis sur le terrorisme international, tous deux demeurant parfaitement cloisonnés grâce à l'aide des médias.

Sur le plan de la politique extérieure, depuis 1972, à l'ONU, où elle fut défendue par George Bush senior, alors ambassadeur, la position officielle des États-Unis (et d'Israël) est de ne pas reconnaître le terrorisme d'État (state-sponsored). Ce à quoi s'opposent les États arabes et, notamment, les Palestiniens qui dénoncent un terrorisme «originel » lié au colonialisme, et justifient leurs actes de violence comme une lutte pour l'autodétermination des peuples. Causes et méthodes trouvent ainsi leur logique, aussi discutable soit-elle. Or les médias ont très rarement relayé la teneur des arguments des Palestiniens et les diverses luttes au sein de l'ONU sur la question des définitions. Du coup, les Américains ne sont pas exposés aux motivations et légitimations de la partie adverse, dans

' Le terme d'« obfuscation » est emprunté à D. Dayan (2005). En français, on pourrait le traduire par celui d'assombrissement. En informatique, l'« obfuscation » consiste à créer une partie de code ou de programme incompréhensible à quiconque n'en possède pas les clés. Pour D. Dayan, le recours à ce procédé rend illisible le contenu informationnel transmis. 
un contexte professionnel où les journalistes sont par ailleurs encouragés à utiliser un script qui décrit le « comment » de la violence et rarement son « pourquoi ». En politique intérieure, les gouvernements américains successifs ont tenu à l'intention du peuple américain un discours dénonciateur de la violence internationale et du terrorisme d'État, avec la production, chaque année, d'une liste d'États « voyous » (rogue states). L'étanchéité des deux discours est telle qu'elle ne fait pas apparaître les contradictions américaines aux Américains et qu'elle ne leur présente pas la situation dans sa complexité, mais dans son manichéisme (us vs them). L'incompréhension du public américain lors des attaques du II-Septembre peut s'expliquer en partie par cette double illusion : que tout le monde pensait comme lui et que tout le monde l'« aimait » (paroles reprises à satiété sur les ondes: « Why don't they like us ? »- Pourquoi ne nous aiment-ils pas ?).

Cette vision des relations internationales héritée de la guerre froide n'a pas beaucoup bougé : elle est relayée par les médias, y compris ceux qui font référence. Elle est à géographie variable et, en vérité, donne au gouvernement américain beaucoup de latitude pour expliquer ses propres interventions dans les affaires d'autres États, avec la croyance fondamentale que leur ingérence se justifie pour le bien de la démocratie et que leurs actes ne peuvent être assimilés à du terrorisme. Toute realpolitik mise à part, ce qui frappe c'est le suivisme des médias américains. La résistance présentée par les Palestiniens est montrée comme irrationnelle, sans fondement et sans relation à une cause nationaliste ou indépendantiste. Les méthodes utilisées sont interprétées comme étant immorales, voire lâches et inhumaines, ce qui peut justifier le recours à des méthodes punitives. De là à associer immoralité à infériorité, il n'y a qu'un pas...

«Tous les langages ne sont pas des novlangues » dit Daniel Dayan (2005 : 198). J'ajouterais : «....Mais ils peuvent tous potentiellement le devenir ». La question de la langue autoritaire peut alors se confondre avec l'autorité des médias. Ici, les mots sont peut-être à interroger autant que les images. Je prendrai deux cas concernant le conflit israélopalestinien tel qu'il est traité aux États-Unis, cas de dénonciation par des journalistes américains assez rares pour mériter d'être mentionnés. Norman Solomon (200I) fait état d'une recherche quantitative sur Nexis, menée sur les 100 premiers jours de l'année 200I, sur toutes sortes de médias. II répertorie plusieurs douzaines d'utilisations de la formule «Israeli retaliation » ou « Israel retaliated » (représailles israéliennes) et une seule occurrence du terme « Palestinian retaliation 》 (représailles palestiniennes). Les deux parties décrivent leur violence comme de l'ordre de la riposte en état de légitime défense, mais seuls les Israéliens voient leurs actes qualifiés de la sorte. Et Norma Solomon (200I) d'expliquer ce parti pris rédactionnel par la menace crédible des 
lobbies pro-Israël, qui dénoncent systématiquement toute couverture favorable à la Palestine comme étant un acte d'anti-sémitisme, créant ainsi une autocensure très efficace, par préemption.

L'observatoire progressiste des médias, Fairness and Accuracy In Reporting (FAIR), conforte cette action des groupes de pression pour éliminer certains mots au profit d'autres, créant de facto une novglangue qui peut se suivre à la trace par sa répétition quasi à l'identique sur divers supports. FAIR analyse avec finesse l'emploi du mot « voisinage » ou « quartier » (neighborhood) destiné à remplacer le mot « colonie » (settlement), dans le contexte du conflit israélo-palestinien, lui-même à recadrer dans le contexte américain où « voisinage » se prête à la double interprétation communautaire et communautariste, toutes deux positivement connotées. II suit la mutation du langage sur CNN, la chaîne de référence pour l'actualité audiovisuelle, qui, en septembre 200I, a annoncé qu'elle changeait sa terminologie, autour du cas de Gilo, une colonie israélienne sur les terres occupées par Israël depuis 1967 : « We refer to Gilo as "a Jewish neighborhood on the outskirts of Jerusalem, built on land occupied by Israel in 1967". We don't refer to it as a settlement $\|^{2}$. CNN cédait en cela à la pression de " CNN Watch, honestreporting.com and other pro-Israeli pressure groups $»^{3}$, selon le reporter spécialiste du Moyen-Orient, Robert Fisk (London Independent, 03/09/0 I). Et de montrer que, même sur la très publique radio nationale, NPR, Gilo est présenté comme faisant partie de Jérusalem, alors que c'est un territoire annexé. Le NewYork Times n'est pas en reste, qui, décrivant les efforts israéliens pour construire des fortifications entre Bethléem et Gilo, présente ce dernier comme un « nearby East Jerusalem neighborhood, where a sprawling Jewish area has been built on land seized after the war of $1967 \gg$.

De cette couverture partiale, il résulte une adhésion du peuple américain à Israël et à sa cause, sans réelle compréhension de la réalité telle qu'elle est vue par la partie adverse. C'est d'autant plus à souligner que les médias commerciaux américains se réclament de la norme d'objectivité et de l'impartialité, ce qui n'est pas le credo des médias français... Une série d'enquêtes menées par le Pew Research Center, entre 2005 et 2006, est assez révélatrice de ces positionnements de l'opinion publique américaine, en comparaison avec l'opinion publique internationale. Dans leur grande majorité (48\%), les Américains soutiennent Israël et la

\footnotetext{
${ }^{2}$ « Nous faisons référence à Gilo comme à "un voisinage juif aux faubourgs de Jérusalem, construit sur un territoire occupé par Israël en 1967". Nous ne nous y référons pas comme à une colonie ».

${ }^{3}$ « CNNwatch, honestreporting.com et d'autres groupes de pression pro-israéliens ».

${ }^{4}$ « Un voisinage proche de Jérusalem Est, où un secteur juif en expansion a été construit sur un territoire saisi après la guerre de $1967 »$.
} 
politique américaine au Moyen-Orient. Par contraste, les Européens apparaissent comme très divisés : une majorité de Britanniques (29\%) et d'Espagnols (32\%) soutiennent la Palestine plutôt qu'lsraël, tandis que les Allemands (37\%) soutiennent plutôt Israël et que les Français sont, pour leur part, à égalité (38 \%-38 \%).

Difficile ensuite de ne pas parler des effets de l'autorité des médias, un point sur lequel je ne suis pas aussi catégorique que nombre de collègues français, dont Daniel Dayan. N'y a-t-il pas une contradiction étrange dans ce déni des effets et cette volonté de critique des médias ? Pourquoi se donner tant de mal à déconstruire leur discours s'il est inefficace et, somme toute, bénin ? Reconnaître les effets ne signifie pas qu'il faille aller à la censure : cela permet au contraire d'aller à la critique, dans toutes ses formes, négatives et positives, constructives et alternatives... Dire qu'il y a des effets ne signifie pas nécessairement que tous se produisent à l'identique, ou qu'ils sont là où on les attend. Stuart Hall (1980) a bien montré que cela pouvait aller de l'adhésion totale entre la source et la cible à la dénégation. Selon lui, la lecture des messages peut être dominante-hégémonique (le public reçoit le message voulu par la production), oppositionnelle (le public n'adhère pas du tout au message), ou négociée (le public fait des ajustements au message).

Dans les cas de violence extrême, le problème des effets tient à ce que ceux-ci se radicalisent avec l'exigence de passage à l'acte politique, c'està-dire au vote ou à la prise de décision d'entrer en guerre ou pas. Comme la limaille de fer sur l'aimant, ils polarisent l'opinion publique, qui doit se manifester en agrégat. Le flou de la boîte noire se résout à la sortie des urnes par une décision sans ambiguïté. La situation de réception est alors essentielle à prendre en compte. Dans le cas du conflit israélo-palestinien aux États-Unis, les médias ont joué sur les mots qui, eux-mêmes, travaillent sur la proximité culturelle et la mise en compatibilité cognitive : les Américains, notamment les évangéliques, se sentent eux-mêmes aussi élus que le peuple élu, partageant avec lui la quête de la Terre promise, ce «voisinage » idéal en projet de construction perpétuelle.

Cherchant à dépasser cette polémique parfois paralysante et pour rendre compte de la complexité des effets associés à des usages, je préfère dire qu'il y a socialisation par les médias, y compris socialisation à des croyances en adéquation avec les attentes générées par une culture donnée. À ce titre, les médias fournissent un environnement culturel englobant, créant un univers de signes cohérents. Et c'est bien cet univers de signes qu'il faut interroger en profondeur, constamment, dans une perspective de cognition située. Celle-ci tient compte de l'interaction entre acteurs et objets et introduit l'histoire et le contexte de l'action. Elle permet, selon Louis Quéré (1987), de penser comment l'action est 
modélisée, en termes de résolution de problèmes, au-delà d'une description mécanique de cause à effet. Elle relativise la place de la seule pensée rationnelle et conceptuelle chez les acteurs sociaux et leurs modes de représentation ; elle redonne de la valeur à l'émotion ainsi qu'à la performance et à la compétence des individus.

\section{La couverture de la guerre et de la terreur}

Ces comparaisons internationales n'ont de valeur que si elles servent à faire émerger des phénomènes récurrents ou des tendances profondes des médias et de la nature humaine. Sans faire de relativisme culturel, et sans chercher à amoindrir la douloureuse spécificité du conflit israélopalestinien, force est de constater que les médias procèdent par obfuscation et détournement dans leur couverture des conflits. Ce schéma me semble généralisable à la couverture de la violence extrême, en temps de guerre, de terreur ou de génocide. II pose la question du rapport à la violence, et partant, de la vision de l'Autre.

J'ai travaillé sur la représentation des violences extrêmes, tels le génocide et la représentation de l'autre étranger, en examinant les cas du Rwanda, de la Bosnie et du Kosovo. Je n'ai pas eu le temps de publier cette recherche, mais toutes les recherches n'ont pas vocation à l'être : elles peuvent rester à l'état d'un journal du chercheur et cette littérature grise sert de matrice à bien des développements ultérieurs, comme un gisement d'idées en attente d'exploitation. Sans adhérer à l'idée que les médias français ont procédé à la création de l'Israélien en « figure radicale de l'inhumanité »(Dayan, 2005 : 202), car le pluralisme des médias français vient quelque peu complexifier cette figure, par contraste avec les médias américains qui angélisent le Palestinien, tout en le « démonisant », force est de constater que la déshumanisation de l'autre étranger est un phénomène récurrent dans les médias. II est à mettre en relation à des processus cognitifs, qui nécessitent la « bestialisation » de l'autre pour pouvoir le tuer sans mauvaise conscience.

La situation au Rwanda ou au Kosovo n'a pas démontré autre chose. En cela, le déni d'humanité ne peut pas se réduire « au retour de la grande tradition de l'antisémitisme théologique » (ibid. : 203). II relève davantage de la barbarie, une action inhumaine qui traite l'autre comme un déchet. Dans Auschwitz et Jérusalem, Hannah Arendt (1948) met en évidence « la logique » qui prévalait dans les camps d'extermination nazis : la mise à mort était décidée ou levée d'une façon tout à fait arbitraire, au hasard de l'humeur des bourreaux. De même, lors de la couverture de guerres et de génocides, un certain nombre d'images télévisées reposent sur la mise en scène d'actes de torture et de gestes de violence gratuite qui rendent banal ce qui devrait rester de l'ordre du 
tabou et de l'inhumain. La barbarie est donc un recours manichéen à des oppositions basiques, qui détruit tous les efforts de la civilisation pour penser le monde dans sa complexité et le réguler par l'État de droit. C'est en cela que l'autorité des médias peut faire peur. Elle réfute toute réflexion sur l'information en temps de guerre. Toute réflexion distanciée sur le caractère lacunaire ou biaisé de l'information dont dispose le public relèverait de la sécheresse de cœur ou d'une insensibilité lâche ou criminelle. Cette autorité se manifeste par le fait que les modes de représentation télévisée de la barbarie et leur usage dans l'information télévisée interpellent l'émotion pour rallier l'opinion. Le terrain des images est pour le moins aussi miné que le territoire des mots. L'utilisation médiatique de cette violence extrême qu'est la barbarie tend à créer des espaces de solidarité « en trompe-l'œil »: elle ravive un sentiment de lien social fondé sur des chocs émotionnels. Les téléspectateurs communient ensemble dans un même territoire, délimité et protégé par la télévision, ce qui « dé-réalise »leurs responsabilités politiques et sociales.

Une de mes recherches, menée avec Sophie Jehel dans le cadre du séminaire de Jacques Sémelin sur la "barbarie" - qui s'est tenu au CNRS en 2000 -, montre que la violence extrême convoque le lien social par la perception qu'elle nie trois conquêtes politiques et sociales - ce qui n'est pas démocratique, ce qui n'est pas de l'ordre de l'humain, ce qui met en place une relation dissymétrique à l'autre -, avec des enjeux de pouvoir, possibles grâce au jeu entre proximité et étrangeté. La violence extrême est perçue comme régressive par rapport aux acquis de l'État de droit, et elle est ressentie comme une violation de divers accords internationaux. La dimension de l'inhumain ou de l'infra-humain, plus subjective, n'en est pas moins liée à la précédente. Elle aussi pose la question de l'État de droit, mais dans sa relation au sentiment de la justice immanente. Sise dans le non-droit, l'« in-juste », la violence extrême donne le sentiment d'une violation de l'ordre civilisé. La dimension de l'autre et du pouvoir, elle aussi subjective, pose l'existence d'une norme sociale, démocratique, par rapport à laquelle la violence extrême devient « a-sociale » et « a-normale », monstrueuse. Elle frôle le tabou, voire la transgression de l'interdit. Les journaux télévisés la représentent en se focalisant surtout sur le corps (ses mutilations, ses humiliations...) ; elle concerne souvent des groupes ethniques ou des minorités tribales, dans des pays lointains. Ces négations sont associées à des discours dont la télévision se fait soit le relais, soit l'expression : la violation des droits provoque un discours de la dénonciation (à distance), la violation de l'ordre civilisé fait s'élever un discours de l'indignation (impuissante) et la violation de l'interdit conduit à un discours utilisant le voyeurisme (inhibant car dégradant le voyeur passif qu'est le téléspectateur). Ces discours tendent à se télescoper et à se contrecarrer l'un l'autre, avec pour résultat une absence d'action et de réaction de la part de l'opinion 
publique qui a pour conséquence moins sa mobilisation que sa désaffection, avec parfois le sentiment d'une participation forcée au franchissement du tabou dans le type d'images choisies et diffusées.

Pour un spectateur (plus encore que pour un lecteur), il est difficile d'être dissident dans ses réactions face à un spectacle partagé en live, qui vise à l'alignement des positions et à la mise en compatibilité des réactions. Ici, le court-circuitage du raisonnement est opéré par le besoin d'appartenir au groupe face à un événement paniquant. Cela peut expliquer des modifications massives de l'opinion du jour au lendemain (une preuve de la radicalisation des effets ?), comme dans le cas du soutien à une intervention militaire en Afghanistan et en Irak, dans un pays par ailleurs largement isolationniste comme les États-Unis, à la suite du IISeptembre. Je suis moins surprise par la xénophobie en temps de guerre que par la suppression de toute pensée dissidente, qui est souvent son corollaire et permet de bâillonner la liberté d'expression. La fragilité des efforts de civilisation semble n'attendre que l'étincelle de la conjoncture historique pour le passage à l'acte violent, qui évite la médiation de la civilisation. Les contradictions de la violence relèvent des injonctions paradoxales. La violence est souvent attribuée à une socialisation interrompue chez l'individu, mais la violence de groupe, elle, semble provenir des forces mêmes de la socialisation. Ces groupes qui s'entretuent au Rwanda, ou ailleurs, entre guerre civile et génocide, frappent par leur capacité d'organisation et de coopération entre eux, et par leur propension à transformer des individus ordinaires en tueurs. Le système de la violence engendre une pression conformiste, et ôte toute peur de punition ou de violation d'un tabou, tel que la mise à mort d'autrui.

Sans aller jusqu'à adopter toutes les conclusions de certains tenants de la biologie culturelle, dont la cognition située est proche, force est de constater que se produit une régression cognitive. Selon Steven R. Quartz et Terrence J. Sejnowski (2002 : 206-207), elle se caractérise par « l'idéation obsédante, la répétition compulsive, la désensibilisation rapide à la violence, l'émoussement de la réponse émotionnelle et l'hyperexcitation, qui agissent ensemble comme une contagion qui s'étend au groupe entier ». Cette régression peut permettre la mise en place d'une reprogrammation du cerveau : la déconnexion entre l'action et la réponse émotionnelle culturellement adéquate est le but recherché. La contagion de groupe élimine la capacité normale de l'individu à considérer l'autre comme un humain aussi. La connexion entre violence collective et idéologie s'en trouverait confirmée, car il faut une « idéation obsédante » pour conduire une population vers le génocide. Et cette idéation obsédante viendrait de notre capacité culturelle contemporaine à réorienter le comportement, non d'une réaction primitive. Des idées culturellement acquises pourraient venir inhiber notre capacité naturelle 
à l'empathie et nous reprogrammer émotionnellement. Malaise dans la civilisation, disait déjà Sigmund Freud (1915), frappé par le retour à la normale des soldats après la Première Guerre mondiale... Part de l'ombre de notre nature humaine, dirais-je, frappée par la sophistication croissante de notre barbarie et l'apathie de nos réactions.

Mes recherches sur les violences extrêmes ne montrent pas autre chose, si ce n'est le rôle des médias comme catalyseurs de ce processus de déshumanisation par idéation, répétition, désensibilisation, émoussement et hyper-excitation, la « schismogenèse » de Gregory Bateson (1972). N'est-ce pas le jeu dangereux auquel joue une chaîne comme Fox, avec l'idéation obsédante de la «war on terror », qui confond sciemment terreur et terrorisme, et qui a maintenu ostensiblement pendant des années en permanence à l'écran les niveaux d'alerte (jaune, orange, rouge), en les activant de temps en temps pour créer de l'hyperexcitation ?

Certains récusent l'argument avant même de lire l'analyse : accuser les médias de faire le jeu de la violence sociale, voire de la violence de groupe, ce serait ouvrir la porte à un retour de la censure. Ne sommesnous pas devant un refus d'assumer notre propre violence et d'ouvrir un débat qui nous obligerait peut-être à interroger le dysfonctionnement de notre sphère publique, le déséquilibre des forces engagées et les termes réels de tout conflit? Un débat qui contraindrait aussi à assumer que la violence du sujet est un des fondements de la conscience morale et sociale. Celle-ci est l'expérience première qui donne naissance au sentiment de culpabilité. C'est son refoulement qui permet la constitution du lien avec l'autre, ou les autres au sein d'une communauté. La reconnaître pleinement permettrait de démontrer qu'elle ne peut en aucun cas constituer un ferment social, ou une valeur à célébrer, puisqu'il s'agit précisément de la part «a-sociale » de l'être humain. Ne pas le faire conduit à la résignation ou à l'exacerbation de l'agressivité qui propose un horizon de destruction et de mort. C'est dans la dialectisation de nos tendances conflictuelles que le sujet peut se construire, pas dans sa dénégation.

La monstration médiatique d'actions individuelles et collectives de générosité du public et les démonstrations d'altruisme semblent venir en « sur-compensation ». Les associations humanitaires se retrouvent avec des dons qui dépassent quatre à cinq fois leurs espérances. Ne sont-ils pas la réaction naturelle à ces insupportables images de souffrance à distance ? L'image exerce son pouvoir immédiat de compassion, qui suscite instantanément le sentiment de la proximité, si nécessaire au fonctionnement télévisuel. À l'émotion personnalisée correspond la charité individualisée, l'émotif entraîne le caritatif. Faut-il y voir un parallèle avec les rituels des peuples dits primitifs qui, après le carnage, 
devaient accomplir des rites expiatoires ? Donner une obole symbolique serait-il de l'ordre du sacrifice ? Pour conclure avec Steven R. Quartz et Terrence J. Sejnowski (2002), ce qui est troublant dans notre comportement récent, si cru alors que nous sommes si civilisés, ce n'est pas tant le recours à des formes anciennes de la barbarie qui relèveraient d'un cerveau reptilien archaïque, mais les formes nouvelles de la barbarie qui peuvent manipuler ou auto-censurer. Notre propre capacité à intérioriser des croyances et des relations humaines - ce qui nous rend fondamentalement humains - peut nous mener à déshumaniser les autres. Et nous convoquons les médias d'information aux mêmes fins, afin qu'ils nous aident à lever collectivement les tabous que nos sociétés avancées ont utilisés pour se fonder, dont l'interdit de tuer, au nom d'un espace vital ou d'une zone d'influence à préserver...

\section{La réversibilité des images et la médiation journalistique}

Là où on attendrait un débat contradictoire, on trouve des contradictions non surmontées, liées par l'émotion, un parti pris émotionnel qui courtcircuite le raisonnement et voudrait finalement faire croire que tout se vaut, s'équivaut. Daniel Dayan (2005 : 203) s'interroge avec raison sur la réversibilité des images. Déconstruire la réversibilité est un premier pas pour comprendre la déréalisation de l'information télévisée et pour s'interroger sur la médiation journalistique. Selon moi, il s'agit cependant moins d'une pathologie de la lisibilité que d'un syndrome de régression cognitive. À la lueur de l'« idéation obsédante », la réversibilité peut contribuer au phénomène de régression cognitive en ce qu'elle opère ce retournement des valeurs et ce court-circuitage des tabous qui permet à un groupe de reconfigurer ses croyances pour modifier son comportement, comme dans le cas d'Israël construisant des murs alors que les Juifs en ont été victimes dans les ghettos et dans les camps de concentration. Peu après que le monde se soit réjoui de la chute du mur de Berlin, le mur est redevenu protecteur alors que la division était jusque là synonyme de drame et de régression.

Dans le flot audiovisuel et dans la navigation à vue du spectateur de chaîne en chaîne, cette propriété de l'image qu'est la réversibilité prête encore plus à confusion. En effet, une image ne se conjugue ni au conditionnel, ni au subjonctif, ni à l'interrogatif, elle ne se modalise pas. Sa charge émotionnelle tient à son présentisme. C'est encore plus le cas quand elle n'est pas accompagnée d'un commentaire (le fameux « No comment »sur EuroNews). Elle peut alors présenter deux points de vue comme s'ils s'équivalaient, comme s'ils étaient interchangeables. C'est par exemple le cas en Irak, à propos de la torture au temps de Saddam 
Hussein et de celle au temps des Américains. Elle établit une sorte de pseudo-neutralité, qui escamote le caractère irréversible de certaines actions.

La réversibilité créée des équivalences trompeuses entre images dont les sources et les motivations sont diverses. Elles deviennent des armes de manipulation et de surenchère, surtout lorsque l'on ne prend pas en compte leur origine de production et les intentions de ceux qui les diffusent. Ces équivalences aplatissent les réelles différences entre régimes qui créent les violences et les tortures. Or, il ne faut pas franchir le pas des équivalences et aller jusqu'à penser que tous sont interchangeables. Les régimes non démocratiques ne sont pas équivalents à ceux qui sont démocratiques, il n'y a pas de réciprocité possible entre eux. II convient bien de distinguer la réversibilité de la réciprocité, ce qui est la confusion opérée par les médias audiovisuels. La réciprocité relève d'un point de vue plus démocratique qui reconnaîtrait, sans chercher à l'écraser, la différence de l'autre. Quant à la réversibilité, elle est problématique car elle crée une élision de la démocratie. Élision non pas au sens de ce qui est supprimé ou absent, mais au sens de ce qui est supposé être présent comme condition légitime de la représentation et comme raison profonde de rejeter la barbarie et la torture en démocratie. Ainsi, en démocratie, l'objet de la représentation journalistique - l'information comme un droit permettant la constitution d'une opinion publique éclairée - est-il impossible à certifier, ce qui mine la démocratie. Celle-ci échappe à notre connaissance, même si c'est par rapport à elle que tout ce que nous voyons se justifie. La réversibilité permet à la représentation télévisée de se donner comme pure représentation, et nous coupe du « je pense » qui, dans un contexte démocratique, doit pouvoir accompagner toute représentation et toute information.

Les risques de ce genre de couverture sont réels car ils peuvent faire basculer l'autorité des médias vers des médias autoritaires. Ce sont les risques qui font passer d'un certain nationalisme à un réel fascisme. L'uniformisation des soldats-héros, leur renoncement à tout intérêt personnel qui n'irait pas dans le sens de leur mission, l'accomplissement de celle-ci par la force, le simplisme de la grille de lecture politique du monde en conflit entre bien et mal, les traits ouvertement bestialisés ou racistes donnés aux « méchants » : autant d'éléments qui suffiraient à déceler la marque d'une tendance fascisante, dirait Umberto Eco, ou alternativement d'une idéation obsédante.

Loin de moi l'idée de suggérer l'idée que les journalistes sont des fascistes ou que le média audiovisuel le soit. Là n'est pas le moindre des paradoxes : au-delà des convictions personnelles des uns et des autres qui se vivent souvent comme des défenseurs de la démocratie -, il faut s'interroger sur la force du dispositif et la fatalité des fausses croyances 
que ce média véhicule (vitesse, scoop, image, concurrence), tout autant que sur les récits qui sont produits et la nature des articulations qui sont proposées aux spectateurs, surtout quand elles convergent en un seul sens. Le discours qui circule à travers ces images de barbarie sert un idéal politique fascisant parce que la glorification systématique de la violence des bons, tout comme celle des méchants, renverse l'idéal démocratique. La logique de production et le recours à des routines journalistiques ou des scripts bien connus font alors violence. Et, comme Daniel Dayan (2005 : 214), je suis fascinée par un autre paradoxe, qui fait qu'à chaque situation spécifique, les médias réagissent par un discours générique. La mécanique qui fait fonctionner le type de récit fondé sur l'affrontement et la mise en scène de la violence est aussi celle qui le déstructure. La logique de l'affrontement de deux camps peut permettre de faire fonctionner des scénarios qui n'ont dans leur détail quasiment pas de sens intelligible. Le non-sens est alors subsumé sous une logique globale (celle de l'affrontement) qui le rend acceptable. Pris dans une logique de série, le sens de l'épisode n'a finalement pas beaucoup d'importance... La couverture au jour le jour de la guerre en Irak par les médias américains, calquée sur un format de télé-réalité, n'a pas eu d'autre effet que la sérialisation, propice à l'émoussement de la réaction émotionnelle.

Ce à quoi cette réversibilité nous alerte, c'est au besoin de toujours travailler à une meilleure démocratie et à une culture de paix, certes, mais aussi à montrer de l'exigence à l'égard de la médiation journalistique. C'est une médiation « contrainte » par la situation d'information : le journaliste doit dès le départ de l'événement procéder à un travail de fictionnalisation, car il arrive après la bataille, littéralement, et se voit dans l'obligation de procéder à une sorte de reconstitution des faits. Raison de plus pour se méfier des images qui lui sont fournies en « ready-made » par les manipulateurs de l'opinion, comme dans le cas des photos et des vidéos de torture et de mise à mort récemment diffusées sur les antennes. Raison de plus pour s'interroger sur l'illusion entretenue d'une information « en temps réel », d'une couverture des événements sur le terrain qui privilégie l'immédiateté pour obtenir l'adhésion de l'opinion à des événements et des faits dont la gravité implique une temporalité ralentie et une compréhension de l'épaisseur de la réalité et de l'histoire.

Certes, il faut mettre des garde-fous à la profession, en invoquant les termes talismaniques d'indépendance, de vérification et de contrevérification des sources, de suivi des conséquences... en un mot de déontologie. Je n'ai pas rencontré un seul journaliste qui professe autre chose. Mais mettre la seule responsabilité de l'information « journalisée », pour reprendre la terminologie de Daniel Dayan (2005: 217 ) sur les journalistes ne suffit pas. Le dispositif qui les encadre est tout aussi responsable et le poids de la responsabilité doit être partagé entre tous les acteurs de l'information dans les conditions actuelles où l'individu 
isolé n'a aucun contrôle sur les infrastructures qui l'emploient. Ce dispositif n'est pas aveugle mais mû par des pressions privatisantes, et il faudrait interroger les motivations des patrons de chaîne, dans un contexte où la propriété des médias n'est pas le fait des professionnels de l'information mais de la défense et du béton, selon le rapport Lancelot ${ }^{5} .$. À qui profite l'idéation obsédante ?

Dans ce contexte où les notions de « public » et de «privé » sont brouillées, difficile de maintenir le sens des valeurs éthiques. C'est peutêtre possible en ayant recours à cette partie du public qui veut rester libre de sa pensée et de ses médias, qui veut du pluralisme, de la tolérance et du lien social. Ce public n'est pas dupe. Mais, même s'il est attentif à ces questions et même s'il est très éduqué, il ne peut faire cette analyse de réversibilité à chaque fois qu'il regarde la télévision. Ce dont il a besoin, outre le recours indispensable à d'autres médias de complément, c'est du recours à d'autres formats que le journal télévisé, comme le magazine ou la revue. À cet égard, il peut militer pour que ces formats ne soient pas éliminés de l'offre audiovisuelle, militer pour qu'il y en ait plus, militer pour que certaines chaînes restent indépendantes et produisent une information de référence. Aux États-Unis, les tortures d'Abu Graïb ont été dénoncées par l'émission magazine 60 minutes sur CBS et par le magazine de presse écrite, The New Yorker (deux longs articles de Seymour Hersh, mai 2004). Le relais a ensuite été pris par le politique et une série d'enquêtes publiques. Ce n'est pas un hasard (et, ajouterai-je, la réciprocité n'a toujours pas eu lieu en Irak). II s'agit bien de préserver avec vigilance un espace public et médiatique où peuvent s'exprimer les contradictions, en œuvrant à une co-responsabilité de tous les acteurs, y compris en faisant appel à la responsabilité sociale des entreprises et des pouvoirs publics. Sans cela, le risque est que la réalité se rebiffe, que la confiance se perde et fasse perdre les avantages acquis pour le bénéfice de tous. Ces dommages collatéraux sont irréversibles.

\section{Événement et situation}

En partie, c'est cette analyse qui me fait dire, un peu par provocation il est vrai, que l'événement n'existe pas, seule la situation lui donne sens. Daniel Dayan (2005 : 197) souligne lui-même que le traitement de la seconde Intifada avait déjà donné lieu à « une répétition générale du

\footnotetext{
${ }^{5}$ En janvier 2006, le Professeur A. Lancelot a remis à D. de Villepin, premier ministre, un rapport traitant des problèmes de concentration dans les médias. On peut y lire que la concentration n'aurait pas atteint un niveau alarmant et que les règles de concurrence dans le secteur des médias se révèlent trop rigides par rapport aux règles communes. Des aménagements ont donc été proposés par la commission ayant en charge cette réflexion. Le rapport est consultable sur le site : www.ddm.gouv.fr
} 
traitement », dans les années 70-80. De même pour le World Trade Center, l'attentat de $1993^{6}$ préfigurait celui de 200I, avec les mêmes acteurs, les mêmes émotions et déjà un nombre significatif de morts (un seul ne suffit-il pas dans ces cas-là ?). Ce qui « crée l'événement » peut tenir à l'ampleur et la radicalité d'un fait historique, mais ce ne sont pas des facteurs explicatifs satisfaisants et suffisants. II faut toute une série de pré-événements, d'événements précurseurs de moyenne intensité qui mettent le public en état de veille, et qui viennent confirmer tous les soupçons quand l'événement « expressif » de forte intensité, comme le dit Dayan Dayan, se produit. Je parlerai plutôt d'événement « mutatif » en y rajoutant une dimension de cognition située : un événement mutatif est de l'ordre de la régression cognitive et permet un changement paradigmatique, à savoir la transformation de l'agenda social et, en conséquence, de l'agenda médiatique. Le recours à l'identique de la situation antérieure est impossible, irréversible. En cela la régression n'est pas nécessairement négative : elle permet de faire le point sur un nouvel arbitraire du monde et de réorienter le traitement de l'information et la nature de l'action attenante.

C'est ce que j'essaie de démontrer dans Qui a détourné le / / septembre? (2006). L'événement mutatif s'inscrit dans un faisceau de faits contradictoires qui lui sont antérieurs et parfois même en sont indépendants : il leur sert de résolution. À la sortie de ce type d'événement s'opère comme une sortie de crise : les paradigmes dominants ne sont plus les mêmes. Ainsi les États-Unis ont-il changé de paradigme avant et après le I|-Septembre : d'une stase paralysante entre politiques d'identité et politiques de moralité, ils sont passés à deux nouveaux paradigmes centraux dans le discours politique, sécurité contre environnement, qui rétablissent des lignes de partition claire entre Républicains et Démocrates. La relation entre identité et moralité avait peu à voir avec le terrorisme international mais beaucoup avec les préévénements expressifs, comme l'élection présidentielle controversée de 2000, l'explosion de la bulle des dot.com7, les scandales financiers et boursiers à répétition autour de l'affaire Enron', etc. De même, les

\footnotetext{
${ }^{6}$ Le 26 février 1993, un premier acte terroriste touchant le World Trade Center avait provoqué la mort de six personnes et blessé plus d'un millier. Ayant subi de graves dommages, les deux tours étaient restées fermées pendant plusieurs mois.

${ }^{7}$ Dotcom ou point com désigne une start-up ou une filiale internet d'un groupe.

${ }^{8}$ Société texane, Enron était l'une des plus grandes entreprises américaines, par ailleurs très bien cotée en bourse. En plus de ses activités dans domaine du gaz naturel, elle avait mis en place un système de courtage, lui permettant d'acheter et de revendre de l'électricité, par exemple au réseau des distributeurs de courant de l'État de Californie. À la fin de l'année 200 I, elle fit faillite en raison des pertes occasionnées par ses opérations spéculatives sur le marché de l'électricité ; des pertes masquées en bénéfices à l'aide de manipulations comptables. Cette faillite provoqua celle d'Arthur Andersen, qui auditait les comptes d'Enron.
} 
politiques de sécurité comme celles de l'environnement ont peu à voir avec le terrorisme international : il s'agit de sécurité au sens de confiance dans les réseaux numériques, dans le marché, dans la politique et d'environnement au sens de climat, énergie, protocole de Kyoto, etc. Certes les questions d'identité et de moralité existent encore, mais elles sont résiduelles, et analysées à travers les nouveaux paradigmes.

C'est là aussi que le discours générique face à une situation spécifique prend une autre dimension. La régression cognitive en situation consiste à placer un fait dans une mise en conformité à d'autres grands récits fondateurs et mnémotechniques. Un événement mutatif ne fait sens et ne reste dans les mémoires comme tel, un trauma transformé en fable, que s'il fait intertexte. C'est alors qu'il acquiert une dimension d'irréversibilité. Tout est fait dans les récits épiques et les mythes fondateurs pour donner aux actes de violence une dimension symbolique, c'est-à-dire à la fois irréversible, fondatrice d'une nouvelle organisation et unique. Ce processus n'échappe pas aux médias, et recoupe les trois fonctions répertoriées par Harold Lasswell (1948 : 325I) : observation (surveillance de l'environnement et de ses risques), corrélation (création du lien social et du consensus par débat d'opinion et persuasion) et transmission (socialisation aux normes et aux croyances d'une culture). J'y ajoute la fonction d'acculturation pour tenir compte de la mondialisation et de la diversité des publics actuels qui ne sont plus seulement nationaux mais internationaux et intra-nationaux, du fait des diasporas multiples. Convaincre ces publics diasporiques de la validité des grands récits fondateurs et mnémotechniques n'est pas le moindre des enjeux de l'internationalisation des médias.

\section{La cognition située comme une contribution au discours de la méthode}

Les catégories de l'expressif et du mutatif posent d'emblée l'intéressante question du corpus. Daniel Dayan montre lui-même à quel point les catégories qu'il a établi avec Elihu Katz (1992) à propos de la télévision cérémonielle (《 coronations, conquests and contests ») sont marquées par le choix du corpus et les bornes temporelles définies. Sur un corpus décalé, James W. Carey peut leur opposer, ou plutôt, y rajouter d'autres notions moins nobles et épiphaniques autour des rituels d'exclusion, d'humiliation et d'excommunication. Ces catégories sont proches de la représentation des violences extrêmes et permettent de réintégrer la régression cognitive et les dimensions de l'idéation obsédante dans

\footnotetext{
${ }^{9}$ « Couronnements, conquêtes et compétitions ».
} 
l'équation médiatique. Aujourd'hui, il faudrait aussi se poser la question du statut des catastrophes naturelles qui ont fait irruption dans notre paysage médiatique pour leur violence extrême. Elles entrent en résonance avec le paradigme de l'environnement dont on n'a pas fini d'évaluer l'importance et d'interpréter les conséquences...

Ma recherche en cognition située se place aussi dans une perspective d'environnement. En effet, je m'inspire de la biologie culturelle pour modéliser la transmission des idées sur celle de la transmission des réseaux neuronaux. II s'agit d'appliquer les théories de l'esprit aux notions d'information et de récit, d'acteurs et de situation. Des auteurs comme Joseph LeDoux (1996), Michael Tomasello (1999), Lawrence Harrison et Samuel P. Huntington (2000) ont établi des parallélismes frappants entre la reproduction sociale de la culture et la reproduction biologique des cellules de notre cerveau. J'essaie d'ajouter la dimension de la communication dans cette équation en me fondant sur l'idée que les médias imitent ces mécanismes dans la reproduction de données à l'ère de l'analogique et du numérique. Les médias sont construits comme des extériorisations des réseaux neuronaux, le cerveau humain étant en co-évolution avec l'environnement biologique. Réciproquement, les médias permettent des intériorisations de la production d'autres réseaux neuronaux, produits par la culture et mis en circulation par les médias, en situation. II ne s'agit pas d'entrer dans les détails chimiques de notre biologie qui sont étudiés par d'autres chercheurs, en neurosciences, mais de voir les incidences que cela a pour les médias en société. Désormais, les avancées des sciences cognitives permettent de passer de l'analyse clinique à l'analyse sociale et politique, avec précaution certes, mais sans atermoiements.

Dans cette perspective, les médias sont des prothèses à mémoires. Ce sont des formes mémorielles de traitement de l'information et de résolution de problèmes, sinon de conflits. Comme la mémoire, ils peuvent procéder à l'oubli, à l'obfuscation, à la sélection, au détournement... Ce sont des artefacts cognitifs non biologiques qui organisent une mémoire collective, partagée, distribuée, autour de grands récits mnémotechniques. Ils viennent soutenir et modifier la mémoire biologique, dont le fonctionnement est limité en stockage. Les représentations qu'ils véhiculent peuvent aussi être internalisées en retour et alimenter la mémoire individuelle, comme par procuration, s'y installant durablement pour nourrir la base des connaissances. D'où l'importance d'être vigilants en ce qui concerne les représentations et particulièrement celles des violences extrêmes, car notre capital social est en partie renouvelé par elles. Elles procèdent à nos cadrages cognitifs socialisés et scellent les valeurs de notre pacte culturel. D'où aussi la nécessité de recherches qui incorporent producteurs et récepteurs dans un continuum où les médias et leurs messages soient évalués comme thèmes privilégiés de l'attention collective. 


\section{Conclusion}

Cette approche en cognition située est peu développée en France. À cet égard, la position de la recherche française reste très timide, sans doute à cause d'un certain ancrage disciplinaire dans les seules sciences humaines, comme le remarque Dayan (2005 :218), sans doute aussi par crainte d'un réveil des fantômes de la censure. II est pourtant essentiel de se confronter à notre existence biologique et médiatique. Ne pas le faire, c'est laisser la place aux paniques ou aux abus. Des vues extrêmes de la nature humaine ou de l'environnement peuvent alors régner sans être remises en cause. Cela peut nous cacher notre véritable nature tout comme celle des médias, à l'interface de la sphère publique et de la sphère privée. Pour éviter tout déterminisme - technologique, biologique ou médiatique -, il faut se plonger dans la complexité et la plasticité sans cesse renouvelées de cette relation média-cerveau. Complexité et plasticité permettent de ne pas la prendre comme une fatalité et, en conséquence, d'essayer d'élaborer des solutions et de proposer des alternatives ou des reconfigurations.

Cette approche permet également de résoudre ces tensions paradoxales que tout chercheur en information-communication éprouve à l'égard des médias, à un moment ou à un autre de ses recherches : la reconnaissance de leur utilité sociale et civique, d'une part, la nécessité de les critiquer avec dureté et bienveillance, d'autre part. Se rappeler qu'ils ne sont somme toute que des prothèses permet de ne pas leur laisser une autorité à laquelle ils n'ont pas droit, par un abus de « faire croire »dans lequel ils se posent en homéostasie avec nous. Face aux médias dont l'utilité sociale n'est pas à remettre en cause, le chercheur a donc pour mission la critique qui est à la fois un interdit de résignation, un droit à l'indignation et un devoir de proposition.

\section{Références}

Allen J. T., Tyson A., 2006, « The U.S. Public's Pro-Israel History. In Mid-East Conflicts, Americans Consistently Side with Israel », PewResearch.org., 19 Jul., (consulté le 09/12/07).

Arendt H., 1948, Auschwitz et férusalem, trad. de l'anglais par A. Guerin, Paris, Deux temps Tierce, 1991.

Bateson G., 1972, Vers une écologie de l'esprit, trad. de l'américain par F. Drosso, L. Lot et E. Simion, Paris, Éd. Le Seuil, 1977.

Brulin R., 2008, Terrorisme et politique étrangère aux États-Unis, 1980-2001, Thèse en Études américaines, université Paris 3-Sorbonne Nouvelle, en cours.

Dayan D., Katz E., 1992, Media Events :The Live Broadcasting of History, Cambridge, Harvard University Press. 
Eco U., 1993, De Superman au surhomme, trad. de l'italien par M. Bouzaher, Paris, Grasset.

FAIR, 2002, « Euphemisms for Israeli Settlements Confuse Coverage », Extra! 7 juil., fair.org (consulté le 09/12/07).

Frau-Meigs D., 2006, Qui a détourné le // septembre ?, Bruxelles/Paris, De Boeck/lna.

Frau-Meigs D., Jehel S., 1997, Les écrans de la violence, Paris, Éd. Economica.

Freud S., 1915, «Considérations actuelles sur la guerre et sur la mort », pp. 25-35, in : Essais de psychanalyse, trad. de l'allemand par A. Bourguignon, Paris, Payot, 1981 .

Hall S., 1980, « Encoding/Decoding » Culture Media, Language, London, Hutchinson.

Harrison L. E., Huntington S. P., 2000, Culture Matters : How Values Shape Human Progress, New York, Basic.

Lasswell H., 1948, "The Structure and Function of Communication in Society », pp. 32-5I, in : Lyman Br., ed., The Communication of Ideas, New York, Harper \& Row.

LeDoux J. E., 1996, The Emotional Brain, New York, Simon \& Schuster.

Quartz S. R., Sejnowski T. S., 2002, Liars, Lovers and Heroes. What The New Brainscience Reveals About How We Become Who We Are, New York, Harper and Collins.

Quéré L., 1997, « La situation toujours négligée ? », Réseaux 85, pp. 18-29.

Solomon N., 200I, « Bias and Fear Tilting Coverage of Israel », 19 avr., ZNet (consulté le 15/0I/07).

Tomasello M., 1999, The Cultural Origins of Human Cognition, Cambridge, Harvard University Press. 